
https://doi.org/10.1007/s00059-020-04994-0 Eingegangen: 19. Mai 2020

Überarbeitet: 2. Juli 2020

Angenommen: 11. August 2020

Online publiziert: 12 . Oktober 2020

(c) Der/die Autor(en) 2020
Herz 2021 - 46 (Suppl 2):S151-S158

\author{
Andreas J. Rieth ${ }^{1,2}$ (D) Christian W. Hamm ${ }^{1,2,3} \cdot$ Christoph Wanner ${ }^{4}$. \\ Veselin Mitrovic ${ }^{1,2} \cdot$ Till Keller $^{1,2,3}$ \\ 'Kerckhoff-Klinik, Abteilung für Kardiologie, Campus Kerckhoff der Justus-Liebig-Universität Gießen, Bad \\ Nauheim, Deutschland \\ ${ }^{2}$ Standort Rhein-Main, Deutsches Zentrum für Herz-Kreislauf-Forschung, Frankfurt am Main, Deutschland \\ ${ }^{3}$ Medizinische Klinik I, Kardiologie, Justus-Liebig-Universität Gießen, Gießen, Deutschland \\ ${ }^{4}$ Abteilung Nephrologie, Universitätsklinikum Würzburg, Würzburg, Deutschland
}

\section{Herzinsuffizienzprotektion bei Patienten mit Diabetes mellitus Typ 2 durch SGLT2-Inhibitoren - Evidenzlage und mögliche Mechanismen}

\section{Systematische Übersicht}

\section{Hintergrund}

Herzinsuffizienz (HI) ist eine der gefürchtetsten Folgeerkrankungen des Diabetes mellitus mit ungünstiger Prognose und erhöhtem Hospitalisationsrisiko. Medikamente zur Behandlung des Diabetes mellitus können einen günstigen, neutralen oder ungünstigen Einfluss auf eine HI ausüben [1]. Die HI-Leitlinien der European Society of Cardiology (ESC) von 2016 enthalten nur wenige Empfehlungen zur antidiabetischen Therapie bei HI: eine Kontraindikation (Klasse III, Evidenzlevel A: Daten aus mehreren randomisierten Studien oder Metaanalysen) für Thiazolidindione und eine IIa-CEmpfehlung (IIa: „sollte erwogen werden“; Evidenzlevel C: Expertenmeinung) für Metformin. Zur HI-Prophylaxe und Prognoseverbesserung wird an anderer Stelle Empagliflozin, ein blutzuckersenkender Inhibitor des Natrium-GlukoseKotransporters 2 („sodium glucose-linked transporter 2“; SGLT2i), empfohlen (Klasse IIa, Evidenzlevel B: Daten aus einer einzelnen randomisierten Studie oder großen nichtrandomisierten Studien; [2]). Diese Wirkstoffklasse erfährt derzeit großes Interesse mit einer Viel- zahl aktueller Publikationen. Während ein Klasseneffekt der SGLT2i mit positiven Auswirkungen auf eine HI von vielen Autoren angenommen wird, ist der Stellenwert beteiligter Mechanismen (u.a. Blutdruck- und Gewichtsabnahme, Natrium- und Flüssigkeitsverlust, verlangsamter Nierenfunktionsverlust, reduzierter oxidativer Stress, verbesserter Metabolismus) unklar [3].

Die vorliegende Arbeit hat sich daher zum Ziel gesetzt, folgenden Fragen nachzugehen:

- Erlauben die vorliegenden Studiendaten Rückschlüsse über im Kontext der HI-Pathophysiologie plausible zugrunde liegende Mechanismen?

- Zeigt die vorhandene Evidenz aus randomisierten, kontrollierten Studien eine Verhinderung von HIEreignissen durch SGLT2i als Klasseneffekt?

- Wie ist das Nutzen-Risiko-Verhältnis durch unerwünschte Arzneimittelwirkungen zu bewerten?

\section{Methoden}

Die vorliegende Übersichtsarbeit wurde vorab in PROSPERO (International Prospective Register of Systematic Reviews) registriert (No. CRD42019122656) und nach den PRISMA(Preferred Reporting Items for Systematic Reviews and MetaAnalyses)-Empfehlungen [4] erstellt. In einer selektiven PubMed-Datenbankrecherche fanden folgende Suchbegriffe Verwendung: Date - Publication: 2009/02/09 to 2019/02/09 AND Language: English AND Title/Abstract: Heart Failure AND Title/Abstract: Cardiovascular AND Title/Abstract: Sodium Glucose Cotransporter 2 Inhibitor AND All Fields: Controlled Trial. Alle Originalarbeiten, die über HI-Ereignisse oder Biomarker mit Bezug zur HI als primären, sekundären oder Sicherheitsendpunkt berichteten oder die eine Subgruppenanalyse unter Einbezug von HI durchgeführt hatten, wurden einer näheren Auswertung unterzogen. Berücksichtigung fanden randomisierte klinische Studien, Beobachtungsstudien und Metaanalysen, sofern weitere außer den bereits einbezogenen Studien analysiert wurden oder wesentliche neue Aspekte zum Vorschein kamen. Bei dem erstellten Datenerfassungsbogen standen Daten zur absoluten Risikoreduktion und zur Anzahl von Behandlungen pro verhindertes Ereignis („number needed to treat", NNT) im Zentrum. Das Bias-Risi- 


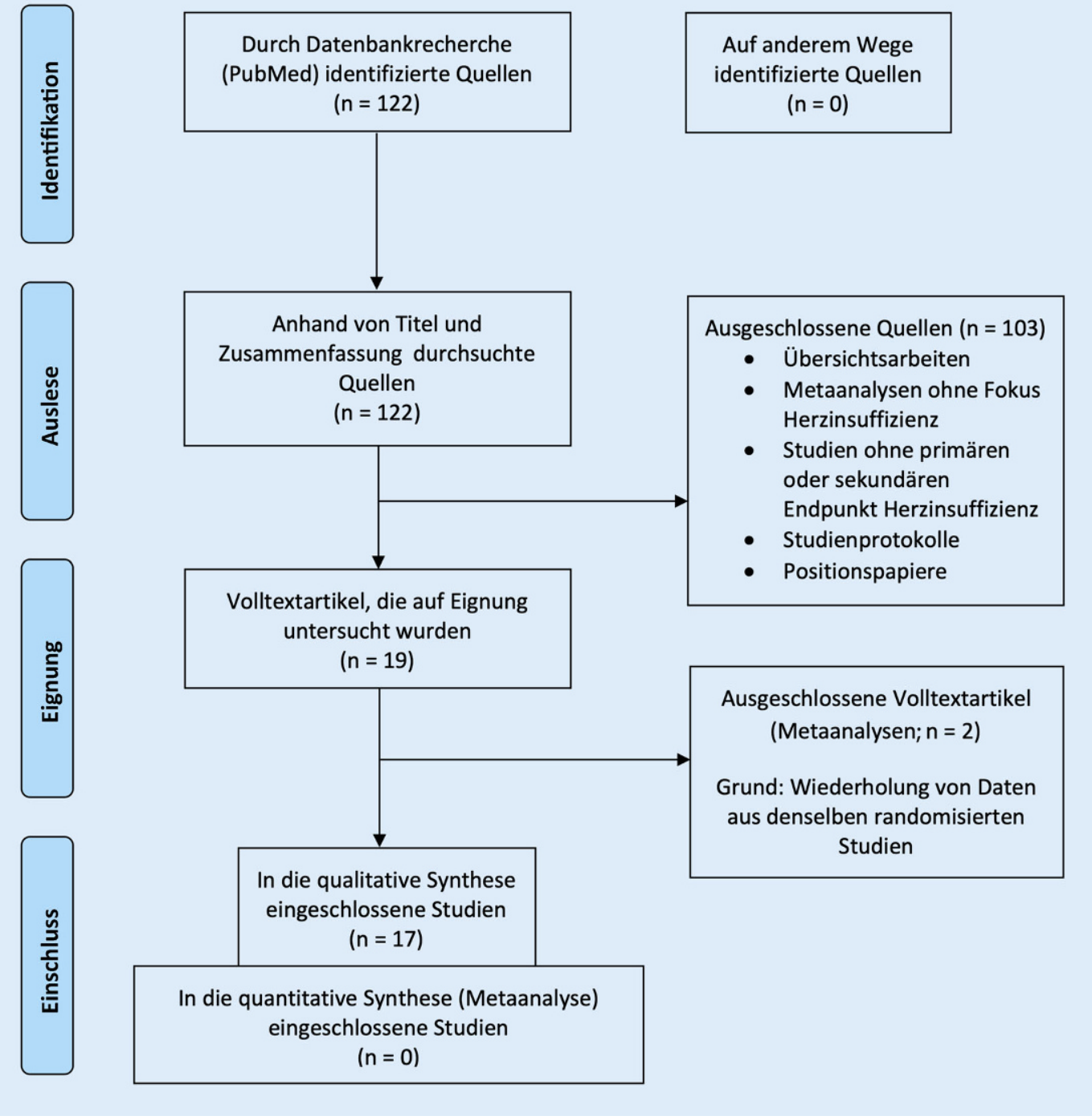

Abb. $1 \triangleleft$ PRISMA(Preferred Reporting Items for Systematic Reviews and Meta-Analyses)-Diagramm: Darstellung des Selektionsprozesses für die in der Übersichtsarbeit verwendeten Quellen [4] ko wurde unter Nutzung des CochraneRoB-2-Tools [5] erfasst, und es wurden nur randomisierte Studien mit einem niedrigen Bias-Risiko eingeschlossen.

\section{Ergebnisse}

Unter den 122 Suchergebnissen fanden sich 9 randomisierte Studien, 10 Posthoc-Analysen, 2 retrospektive Beobachtungsstudien, 32 Metaanalysen, 12 Studienprotokolle/Vorstellungen des Studienaufbaus, 54 Übersichtsartikel und 3 Leitlinien/Positionspapiere.

Von einer weiteren Analyse ausgeschlossen wurden Übersichtsartikel, Leitlinien/Positionspapiere und Vorstellungen des Studienaufbaus; weiterhin alle Studien ohne HI-Endpunkte und $\mathrm{Me}$ taanalysen ohne Fokus auf HI. Ferner wurden 2 Metaanalysen ausgeschlossen, deren Datenbasis bereits Grundlage anderer eingeschlossener Analysen war (- Abb. 1). So kamen letztlich 4 randomisierte Studien, 2 Beobachtungsstudien, 1 Post-hoc-Analyse und 10 Metaanalysen zur Auswertung.

Die größten randomisierten Studien EMPA-REG OUTCOME (Empagliflozin Cardiovascular Outcome Event Trial in Type 2 Diabetes Mellitus Patients; Prüfsubstanz: Empagliflozin), CANVAS (Canagliflozin Cardiovascular Assessment Study; Canagliflozin) und DECLARETIMI 58 (Dapagliflozin Effect on Cardiovascular Events - Thrombolysis in Myocardial Infarction 58; Dapagliflozin) prägten alle evaluierten Metaanalysen entscheidend.

\section{Randomisierte klinische Studien}

Für die 3 SGLT2i-Wirkstoffe aus den oben genannten randomisierten Studien steht mit insgesamt über 34.000 Studienteilnehmern eine umfangreiche Datenbasis zur Verfügung.

In EMPA-REG OUTCOME (2015) wurden erstmals kardiovaskulär bedingter Tod und Hospitalisation wegen HI unter einer Therapie mit Empagliflozin im Vergleich zu Placebo bei 7020 Patienten mit Typ-2-Diabetes mellitus untersucht [6]. Eine HI hatten anamnestisch 9,90\% der Patienten in der Verum- und 10,50\% in der Placebogruppe. Eine geschätzte glomeruläre Filtrationsrate (eGFR) von weniger als $30 \mathrm{ml} / \mathrm{min} / 1,73 \mathrm{~m}^{2}$ war Ausschlusskriterium; eine eGFR von weniger als $60 \mathrm{ml} / \mathrm{min} / 1,73 \mathrm{~m}^{2}$ hatten aber $26 \%$ 
der Studienteilnehmer, sodass hier unter allen randomisierten Studien der Anteil niereninsuffizienter Patienten am größten war. Neben einem positiven Effekt auf den kombinierten primären Endpunkt kardiovaskuläre Sterblichkeit/Myokardinfarkt/Schlaganfall zeigte sich auch ein protektiver Effekt von Empagliflozin auf den sekundären Endpunkt HI-bedingte Hospitalisation. Bei insgesamt relativ geringer Häufigkeit waren Hospitalisationen wegen HI unter Empagliflozintherapie signifikant seltener als unter Placebo (2,70 vs. $4,10 \%$, absolute Risikoreduktion: 1,40; NNT: 72; relatives Risiko [HR]: 0,65 [95\%KI: 0,50-0,85]; $p=0,002$ ). Mit diesem (fokussiert dargestellten) Ergebnis hatte erstmals ein orales Antidiabetikum eine Verbesserung „harter“ kardiovaskulärer Endpunkte gezeigt. Begleiteffekte ohne Signifikanzberechnung in EMPAREG OUTCOME waren eine stärkere Gewichtsabnahme ( $-2,9$ vs. $-1,8 \mathrm{~kg})$ und eine Reduktion des systolischen Blutdrucks $(-2,0$ vs. $0 \mathrm{~mm} \mathrm{Hg})$ unter Empagliflozin $25 \mathrm{mg}$ gegenüber Placebo. Unerwünschte Arzneimittelwirkungen traten in beiden Gruppen insgesamt vergleichbar häufig auf (Empagliflozin: 90 [20\%], Placebo: 91 [70\%]). Die Rate an Genitalinfektionen war dabei unter Empagliflozin höher (6,40 vs. $1,80 \% ; p<0,001)$, schwere unerwünschte Arzneimittelwirkungen jedoch seltener (23,50\% vs. $25,40 \%$; $p<0,05$; - Abb. 2 ).

In CANVAS (2017) wurden kardiovaskulärer Tod, Myokardinfarkt und Schlaganfall als zusammengesetzter primärer Endpunkt unter Canagliflozin bei 10.142 Patienten versus Placebo analysiert [7]. Nur für die Population der Teilstudie CANVAS-R (5812 Patienten) werden explizite Daten zu Hospitalisationen wegen HI berichtet. Von diesen Patienten mit Diabetes mellitus Typ 2 hatten $14,4 \%$ anamnestisch eine $\mathrm{HI}$, bei 20,1\% lag die eGFR zwischen 30 und $60 \mathrm{ml} / \mathrm{min} / 1,73 \mathrm{~m}^{2}$. Der kombinierte sekundäre Endpunkt aus kardiovaskulärem Tod und HI-bedingten Hospitalisationen unterschied sich nur in Letzteren signifikant zugunsten von Canagliflozin (HR: 0,72; $95 \%$-KI: 0,55-0,94; $p=0,0148)$. Die Ereignisrate, bezogen auf 1000 Patientenjahre, betrug 15,85 unter Canagliflozin

Herz 2021 - 46 (Suppl 2):S151-S158 https://doi.org/10.1007/s00059-020-04994-0

(c) Der/die Autor(en) 2020

A. J. Rieth · C. W. Hamm · C. Wanner · V. Mitrovic · T. Keller

Herzinsuffizienzprotektion bei Patienten mit Diabetes mellitus
Typ 2 durch SGLT2-Inhibitoren - Evidenzlage und mögliche
Mechanismen. Systematische Übersicht

Zusammenfassung

Hintergrund. Hemmstoffe des renalen Natrium-Glukose-Kotransporters 2 (,sodium glucose-linked transporter 2"; SGLT2i) scheinen außer der antidiabetischen auch eine kardioprotektive Wirkung zu besitzen; deren Mechanismus ist jedoch unklar. Methoden. Selektive Literaturrecherche in PubMed (Fokus Herzinsuffizienz und Wirkmechanismen).

Ergebnisse. Unter Therapie mit 3 der untersuchten Substanzen kam es im Vergleich mit Placebo zu weniger herzinsuffizienzbedingten Krankenhausaufenthalten, allerdings mit einer relativ hohen Anzahl von Behandlungen pro verhindertes Ereignis (72-117). Außer einer stärkeren Gewichtsabnahme und Blutdrucksenkung unter dem Verum gegenüber Placebo fiel eine Zunahme des kardioprotektiven Effekts bei stärker eingeschränkter Nierenfunktion auf. Schlussfolgerung. Ein moderater herzinsuffizienzpräventiver Effekt von Hemmstoffen des renalen SGLT2 kann bei Diabetikern als gesichert gelten. Ein wesentlicher Wirkmechanismus beruht wahrscheinlich auf einem nephroprotektiven Effekt mit Modulation der kardiorenalen Interaktion, was jedoch weiterer Abklärung bedarf.

\section{Schlüsselwörter}

Empagliflozin · Canagliflozin · Dapagliflozin . Kardioprotektiver Effekt · Kardiorenale Interaktion

\section{Heart failure protection by SGLT2 inhibitors in patients with type 2 diabetes mellitus: evidence and possible mechanisms. A systematic review}

\section{Abstract}

Background. Renal sodium-glucose cotransporter-2 (SGLT2) inhibitors seem to have a cardioprotective effect beyond the antidiabetic effect. The underlying mechanisms are unclear.

Methods. Selective search in PubMed with a focus on heart failure endpoints and possible mechanisms of action.

Results. During treatment with three of the substances analyzed, there were fewer hospitalizations for heart failure compared with placebo; however, the numbers needed to treat within the primary analyses were relatively high (72-117). We found that loss of weight and lowering of blood pressure were more pronounced during treatment with verum than with placebo and an association of the preventive effect with more severely impaired renal function.

Conclusion. The SGLT2 inhibitors show a moderate heart failure protective effect in diabetic patients. It is likely that a nephroprotective effect with modulation of the cardiorenal interaction is an important part of the mechanism of action but this must be substantiated in further investigations.

\section{Keywords} Empagliflozin · Canagliflozin · Dapagliflozin . Cardioprotective effect · Cardiorenal interaction und 21,91 unter Placebo (NNT wegen fehlender absoluter Zahlen unberechenbar). Körpergewicht (-3,9 vs. $-1,6 \mathrm{~kg})$ und systolischer Blutdruck ( $-5,2$ vs. $-2,5 \mathrm{mmHg}$ ) nahmen unter Canagliflozin signifikant stärker $a b$ als unter Placebo. Unter Canagliflozin waren die unerwünschten Arzneimittelwirkungen osmotische Diurese/Volumendepletion 1,4fach und die Zahl peripherer Am- putationen fast 2 fach signifikant erhöht. Die Studienabbruchrate lag unter Canagliflozin bei $29,20 \%$ im Vergleich zu 29,90\% unter Placebo. In dieser Untersuchung konnte bei auch hier niedriger Ereignisrate der in EMPA-REG OUTCOME gesehene positive Effekt auf HIbedingte Hospitalisationen reproduziert werden (• Abb. 2). 


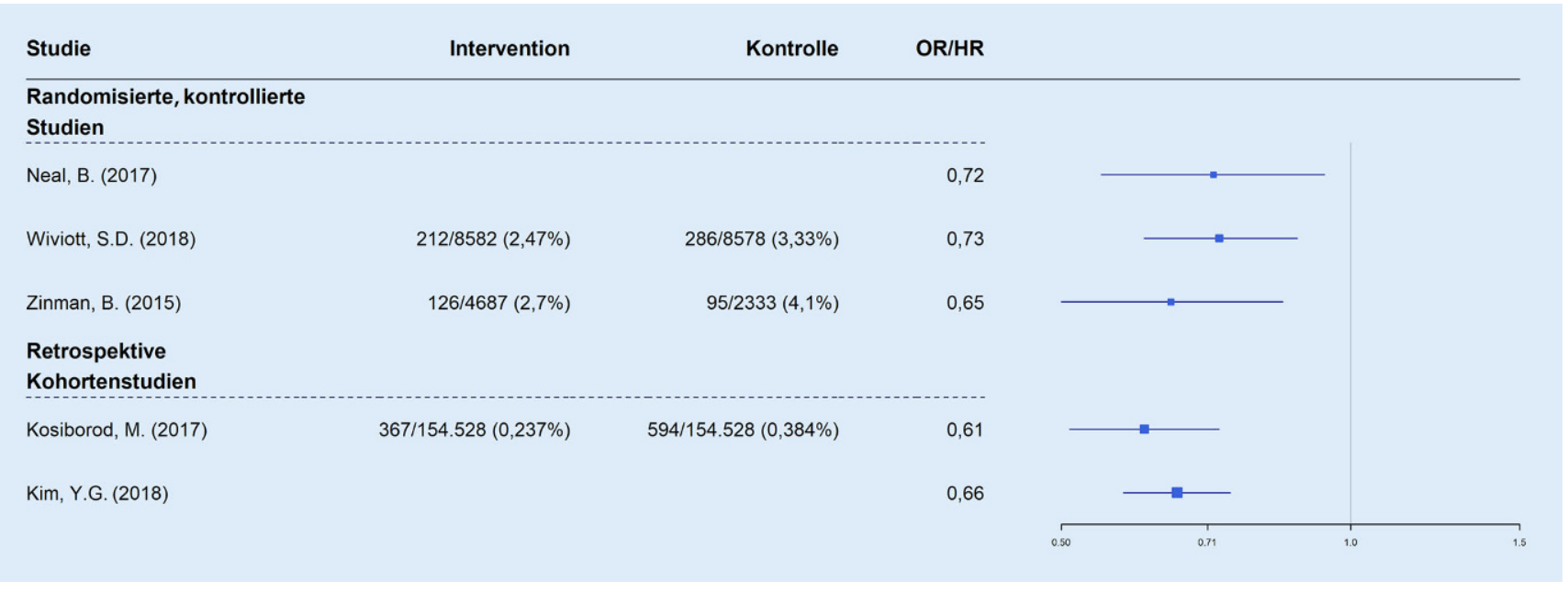

Abb. 2 \ Ergebnisse bisher dargestellter Studien als Forest-Plot: Die Studienergebnisse von EMPA-REG OUTCOME (Empagliflozin Cardiovascular Outcome Event Trial in Type 2 Diabetes Mellitus Patients), CANVAS (Canagliflozin Cardiovascular Assessment Study), DECLARE-TIMI 58 (Dapagliflozin Effect on Cardiovascular Events - Thrombolysis in Myocardial Infarction 58), CVD-REAL (Comparative Effectiveness of Cardiovascular Outcomes in New Users of SGLT-2 Inhibitors) und einer weiteren retrospektiven Studie werden in Bezug auf die Risikoreduktion für Herzinsuffizienzereignisse durch die jeweils untersuchten SGLT2(,sodium glucose-linked transporter 2")-Hemmer dargestellt. Je weiter der Wert unter 1 liegt, desto ausgeprägter ist die Risikoreduktion. OR Odds Ratio, HR Hazard Ratio

DECLARE-TIMI 58 (2018) ist mit über 17.160 Teilnehmern die größte der 3 randomisierten Studien [8]. Eine HI war bei $9,90 \%$ der Teilnehmer in der Verum- und bei $10,20 \%$ in der Placebogruppe vorbekannt, bei $7,00 \%$ von ihnen lag die eGFR bei der Randomisierung unter $60 \mathrm{ml} / \mathrm{min} / 1,73 \mathrm{~m}^{2}$ (eigentlich Ausschlusskriterium). HI-bedingte Hospitalisationen als Bestandteil des primären Effektivitätsendpunkts waren unter Dapagliflozin seltener als unter Placebo (2,47 vs. $3,33 \%$; absolute Risikoreduktion: 0,86; NNT: 117; HR: 0,73 [95\%-KI: 0,61-0,88]; $p=0,005$ für die Kombination aus HI-bedingter Hospitalisation und kardiovaskulärem Tod, nur ersteres unterschiedlich). Nach $48 \mathrm{Mo}-$ naten zeigten sich unter Dapagliflozin eine stärkere Gewichtsabnahme $(-4,00$ vs. $-2,00 \mathrm{~kg})$ und Reduktion des systolischen Blutdrucks $(-3,00$ vs. $0,00 \mathrm{~mm} \mathrm{Hg})$ als unter Placebo. Der kombinierte renale Endpunkt ( $\geq 40 \%$ eGFR-Abnahme auf $<60 \mathrm{ml} / \mathrm{min} / 1,73 \mathrm{~m}^{2}$, terminale Niereninsuffizienz oder Tod renaler Ursache) trat unter dem Verum seltener auf als unter Placebo (4,30 vs. $5,60 \%$; HR: $0,76$ [95\%-KI: $0,67-0,87]$; $p$ : nicht angegeben). Ketoazidosen waren häufiger (0,30\% vs. $0,10 \%$; HR: 2,18 [95\%-KI: $1,1-4,3] ; p=0,02)$, ebenso genitale Infektionen ( $0,90 \%$ vs. $0,10 \%$, HR: 8,36 [95\%-
KI: 4,19-16,68]; $p<0,001)$. Die Abbrecherquote lag aber unter Dapagliflozin niedriger als unter Placebo $(21,10 \%$ vs. $25,10 \%)$. Insgesamt zeigte die Studie einen risikomindernden Effekt von Dapagliflozin auf HI-bedingte Hospitalisationen bei hoher NNT (• Abb. 2).

Eine kleinere randomisierte Studie, die Dapagliflozin versus Placebo an 922 Diabetes-mellitus-Typ-2-Patienten untersuchte, wurde 2015 publiziert [9]. Bei kardiovaskulär Vorerkrankten mit einer eGFR von $60 \mathrm{ml} / \mathrm{min} / 1,73 \mathrm{~m}^{2}$ oder darüber wurde HI lediglich als Sicherheitsendpunkt untersucht. Schwere unerwünschte Arzneimittelwirkungen in Bezug auf HI waren dabei nicht unterschiedlich, jedoch gab es mehr unspezifische kardiale unerwünschte Arzneimittelwirkungen in der Dapagliflozin- als in der Placebogruppe (15,40 vs. $12,50 \%)$, entsprechend einer Anzahl von Behandlungen pro schädigendes Ereignis von 34,48. Hypotension/Dehydratation oder Hypovolämie trat unter Dapagliflozin deutlich häufiger auf als unter Placebo (2,80 vs. $0,40 \%$ ), entsprechend einer Anzahlvon Behandlungen pro schädigendes Ereignis von 42. Die Studienabbruchrate war gering (Dapagliflozin: 12,39\%; Placebo: $12,55 \%)$. Nach 24 Wochen kam es unter dem Verum zu einer stärkeren Gewichtsabnahme $(-2,56 \%$ vs. $-0,30 \%$; $p<0,0001)$ und Senkung des systolischen Blutdrucks $(-2,0 \mathrm{~mm} \mathrm{Hg} ;-2,99 \%$ vs. $-1,03 \% ; p<0,05)$ als unter Placebo (• Abb. 2).

\section{Retrospektive Beobachtungsstudi- en und Post-hoc-Analysen}

2 retrospektive Beobachtungsstudien [10, 11] verglichen, basierend auf Gruppen mit vergleichbarem Risiko, verschiedene SGLT2i (hauptsächlich Dapagliflozin und Canagliflozin) mit anderen oralen Antidiabetika. Insgesamt wurden die Verläufe von 428.014 Patienten u. a. mit dem Endpunkt HI-bedingte Hospitalisation untersucht, dieser ereignete sich in beiden Studien unter dem jeweiligen SGLT2i signifikant seltener als unter anderen oralen Antidiabetika. In der größeren Studie mit 309.056 Patienten (prozentuale Expositionszeit: 52,70\% für Canagliflozin, $42,00 \%$ für Dapagliflozin, 5,50 \% für Empagliflozin) kam es unter SGLT2i seltener zu einer HI-bedingten Hospitalisation ( 0,237 vs. $0,384 \%$; absolute Risikoreduktion: 0,147; NNT: 681; HR: 0,61 [95\%-KI: 0,51-0,73]; $p<0,001$; [10]). In der zweiten Studie (90,2\% Dapagliflozin) lag die Rate der HI-bedingten Hospitalisationen unter SGLT2i ebenfalls niedriger als unter Hemmstoffen der Dipeptidylpeptidase 4 (DPP-4-Hemmer; 


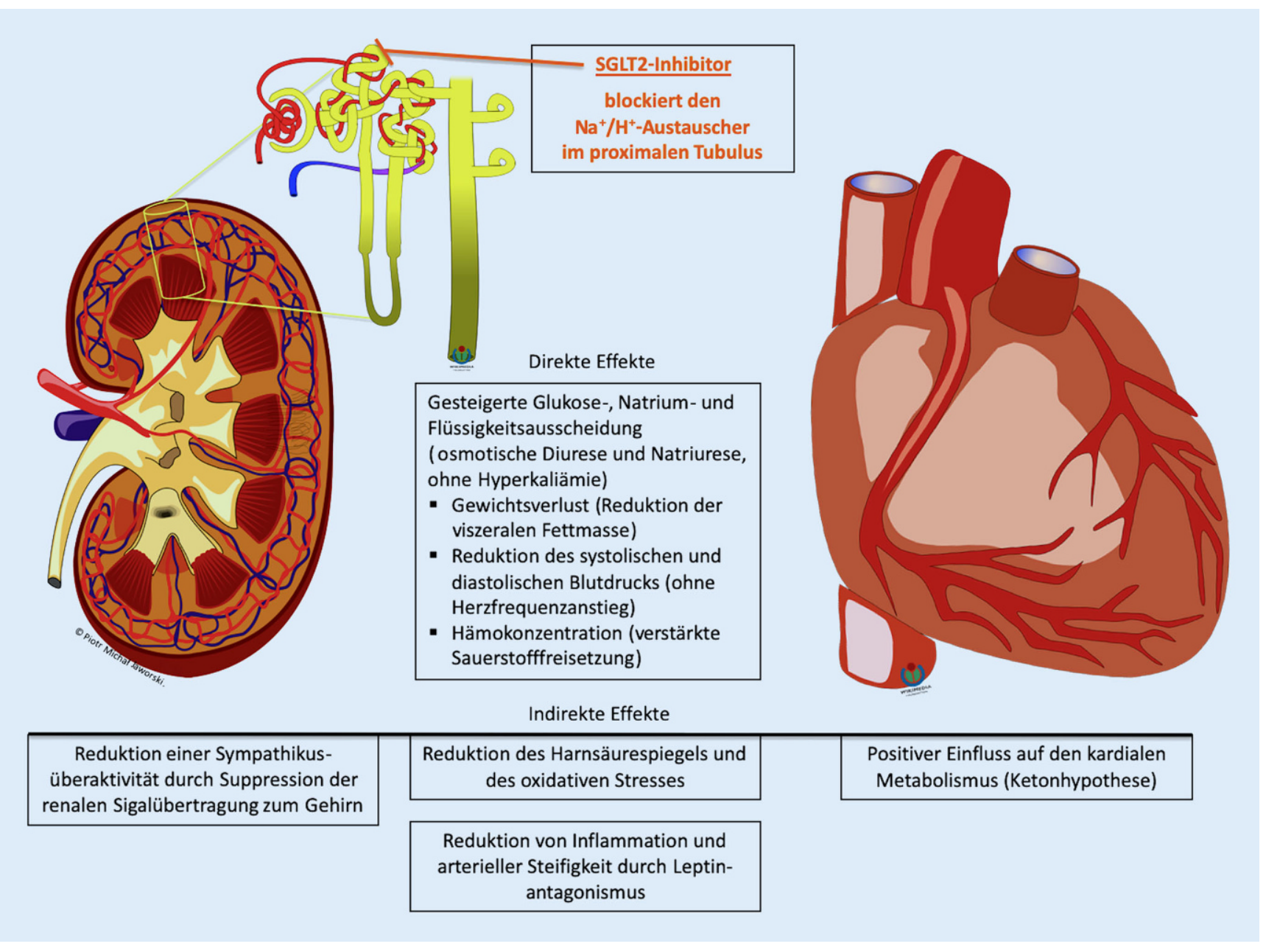

Abb. 3 ॥ Pharmakologische Effekte der SGLT2(,sodium glucose-linked transporter 2“)-Inhibitoren: Dargestellt sind direkte renale Effekte sowie systemische Wirkungen, die teilweise hypothetisch und von unklarer Bedeutung sind

0,83 vs. 1,13 Ereignisse/100 Personenjahre; absolute Risikoreduktion/NNT: nicht berechenbar; HR: 0,66 [95\%-KI: $0,58-0,75] ; p<0,001 ;[11])$. In beiden Untersuchungen war die Zahl der Hospitalisationen wegen $\mathrm{HI}$ sehr niedrig (0,31 bzw. 0,86\%).

Eine Post-hoc-Analyse einer randomisierten Studie mit Canagliflozin versus Placebo mit 666 Teilnehmern untersuchte die Entwicklung von Biomarkern unter SGLT2i-Therapie [12]. U. a. wurde der HI-spezifische Marker NT-proBNP (N-terminales Propeptid „brain natriuretic peptide“) analysiert, der unter erhöhter myokardialer Wandspannung ansteigt. In beiden Behandlungsgruppen kam es nach 2 Jahren $\mathrm{zu}$ einem Anstieg des NTproBNP-Medians; dieser war unter Canagliflozin statistisch signifikant geringer als unter Placebo (2,4 vs. $12,5 \mathrm{pg} / \mathrm{ml} ; p<0,01)$. Dabei lagen aber die absoluten NTproBNP-Werte im Median unter Canagliflozin höher als unter Placebo $(47,4 \mathrm{pg} / \mathrm{ml}$ vs. $43,4 \mathrm{pg} / \mathrm{ml})$, beide im unteren Normbereich [2].

\section{Metaanalysen}

Die untersuchten Metaanalysen sind von den 3 oben genannten großen randomisierten Studien dominiert. 9 von 10 ergaben eine positive Auswirkung von SGLT2i auf HI. Diejenige Metaanalyse mit vorbestehender HI als Einschlusskriterium erbrachte kein signifikantes Ergebnis zugunsten von Dapagliflozin [13]. 3 andere Metaanalysen zeigten einen positiven Effekt von Empagliflozin, nicht aber anderer SGLT2i in Bezug auf HI, die hier über Hospitalisation wegen HI hinausgehend definiert wurde [14-16]. In einer weiteren Analyse wurden nur Patienten mit einer eGFR von weniger als
$60 \mathrm{ml} / \mathrm{min} / 1,73 \mathrm{~m}^{2}$ untersucht; die eGFR lag in den einbezogenen Studien im Bereich von im Mittel 38,00 bis im Mittel $53,50 \mathrm{ml} / \mathrm{min} / 1,73 \mathrm{~m}^{2}$ [17]. Die Untersuchung des HI-Endpunkts (HI-bedingte Hospitalisation oder „fatale $\mathrm{HI}^{\prime}$ ) erfolgte in Bezug auf Empagliflozin, Canagliflozin und Dapagliflozin. Die Rate an HIEreignissen war mit 3,79\% die höchste aller hier präsentierten Studien, und sie war unter SGLT2i niedriger als unter der jeweiligen Vergleichstherapie aus Placebo oder aktivem Komparator (absolute Risikoreduktion: 2,43\%, NNT: 42 , HR: 0,61 [95\%-KI: 0,48-0,78]; p: nicht angegeben). In weiteren Studien zeigten sich positive Auswirkungen der SGLT2i (dominierend: Empagliflozin, zumeist gegen Placebo getestet) auf HI-Ereignisse mit NNT-Werten von $72,84,91$ und 625 [18-21]. Und schließlich erschien eine Metaanalyse der 3 großen 
randomisierte Studien, die das gesamte Patientenkollektiv, aufgeteilt nach eGFRSubgruppen, nachuntersuchte [22]. Die Patienten mit einer eGFR von weniger als $60 \mathrm{ml} / \mathrm{min} / 1,73 \mathrm{~m}^{2}$ profitierten am stärksten in Bezug auf die Reduktion von HI-bedingten Hospitalisationen (relativ: $40 \%$, absolute Risikoreduktion: nicht verfügbar). Lag die eGFR zwischen 60 und $90 \mathrm{ml} / \mathrm{min} / 1,73 \mathrm{~m}^{2}$, betrug die relative Risikoreduktion $31 \%$. In der Gruppe mit einer eGFR von $90 \mathrm{ml} / \mathrm{min} / 1,73 \mathrm{~m}^{2}$ oder darüber ließ sich kein signifikanter Effekt darstellen.

\section{Diskussion}

Seit Publikation der EMPA-REG-OUTCOME-Studie ist es $\mathrm{zu}$ einem starken Interesse an dem beobachteten „AntiHerzinsuffizienz-Effekt" gekommen, als dessen Grundlage eine Vielzahl pharmakologischer Effekte der SGLT2i vermutet werden ([23]; - Abb. 3). Diese Wirkungen hängen teilweise direkt mit deren Hemmung der Natrium-Glukose-Reabsorption im proximalen Tubulus zusammen, die zu einer Natriurese und einer osmotischen Diurese durch Glukosurie führt. Dadurch entsteht ein diuretischer Effekt, der zu Volumenreduktion, Gewichtsabnahme und Blutdrucksenkung führt. Die Mechanismen, durch welche die vielfach belegte nephroprotektive Wirkung der SGLT2i zustande kommt, werden als „wahrscheinlich multifaktoriell“ beschrieben, mit einer vermutlich wichtigen Rolle renovaskulärer Effekte. Eine Aktivierung der tubuloglomerulären Rückkopplung mit Senkung des intraglomerulären Drucks und Reduktion der Hyperfiltration wurde experimentell unter Empagliflozin beobachtet $[24,25]$. Darüber hinaus werden verminderter oxidativer Stress und Verbesserung des kardialen Metabolismus diskutiert. Eine Hypothese besagt, dass die osmotische Diurese unter SGLT2i eine Volumenreduktion im Interstitium unter Aufrechterhaltung des notwendigen intravaskulären Volumens bewirkt. Eine HI könnte hierdurch günstiger beeinflusst werden als unter einer Diuretikatherapie, die bevorzugt das intravaskuläre Volumen reduziert [26]. In einer Mediationsanalyse (statistisches Verfahren, das Kausalität und zeitliche Abfolge zwischen verschiedenen Variablen darstellen soll) von EMPA-REG OUTCOME wurde der Anstieg von Hämatokrit und Hämoglobin als wichtigster Mediator der kardiovaskulären Risikoreduktion von Empagliflozin gegenüber Placebo ermittelt [27], was für den positiven Effekt einer Reduktion des Plasmavolumens spricht. Daten der DAPA-HF(Dapagliflozin And Prevention of Adverse Outcomes in Heart Failure)-Studie [28], die im November 2019 (und damit erst nach der Datenbankrecherche für diese Übersicht) publiziert wurde, ist jedoch zu entnehmen, dass die Behandlung mit Dapagliflozin zu keiner wesentlichen Änderung des Diuretikaregimes geführt hat. Demnach konnte ein "diuretikasparender Effekt" von SGLT2i in der klinischen Anwendung bisher nicht belegt werden.

In allen referierten Studien waren signifikante Körpergewichts- und Blutdruckreduktionen unter SGLT2i zu verzeichnen, wobei sich diese Abnahme beim Gewicht zwischen -2,9 und-4kg, beim systolischen Blutdruck zwischen $-2,0$ und $-5,2 \mathrm{mmHg}$ bewegte. Dieser geringe Unterschied allein reicht zur Erklärung des verringerten HI-Risikos vermutlich nicht aus. In anderen Studien war eine deutlich höhere Blutdrucksenkung zur Senkung des HI-Risikos notwendig. In der SPRINT(Systolic Blood Pressure Intervention Trial)-Studie mit Hypertonikern waren dies $-20 \mathrm{~mm} \mathrm{Hg}$ in der Gruppe mit intensivierter Therapie versus $-8 \mathrm{mmHg}$ unter Standardtherapie [29]. Um einen HI-protektiven Effekt durch Gewichtsabnahme bzw. Reduktion des systolischen Blutdrucks nachzuweisen, wären Subgruppenanalysen notwendig, die bisher nicht publiziert sind.

Die Datenlage $\mathrm{zu}$ Nierenfunktion und HI-Protektion ist besser: Mehrere Arbeiten zeigen Hinweise auf eine ausgeprägtere HI-Risiko-Minderung bei stärker eingeschränkter Ausgangsnierenfunktion. Bei bekannter Interdependenz dieser beiden Organsysteme ist die Niereninsuffizienz eine gravierende Komplikation bei fortgeschrittener HI. Demnach könnte eine SGLT2i-Therapie über Nephroprotektion zu besserer Volumenhomöostase und $\mathrm{zu}$ geringe- rer neurohumoraler Aktivierung führen [30]. Bei einer manifesten HI sind diese beiden Regelkreise gestört [2]. Weiterhin könnte sich die Verhinderung/ Progressionshemmung einer diabetischen Kardiomyopathie [1] durch eine verbesserte glykämische Kontrolle günstig auswirken. Allerdings war die Rate manifester ischämischer Ereignisse in EMPA-REG OUTCOME nicht signifikant unterschiedlich.

Die oben genannte DAPA-HF-Studie konnte einen symptomatik- und prognoseverbessernden Effekt von Dapagliflozin in einem Hochrisikokollektiv (HI-Patienten mit LVEF [linksventrikuläre Ejektionsfraktion] <40\%) unabhängig von einer Diabeteserkrankung nachweisen $(\mathrm{NNT}=21)$. Damit kann ein HI-spezifischer Effekt der SGLT2i als erwiesen gelten, ohne dass mit dieser Studie über die zugrunde liegenden Mechanismen mehr bekannt geworden wäre.

Weitere, aktuell laufende Studien wie EMPEROR (-Reduced [Empagliflozin Outcome Trial in Patients With Chronic Heart Failure With Reduced Ejection Fraction], EudraCT-Nr. 2016-00228034, und -Preserved [Empagliflozin Outcome Trial in Patients With Chronic Heart Failure With Preserved Ejection Fraction], Nr. 2016-002278-11), DELIVER (Dapagliflozin Evaluation to Improve the Lives of Patients With Preserved Ejection Fraction Heart Failure; EudraCT-Nr. 2018-000802-46) und SOLOIST-WHF (Effect of Sotagliflozin on Cardiovascular Events in Patients With Type 2 Diabetes Post Worsening Heart Failure; EudraCT-Nr. 2017-003510-16) bei Patienten mit diabetischer und nichtdiabetischer HI und einem primären HIEndpunkt werden vielleicht auch Aussagen darüber zulassen, ob die Wirkung der SGLT2i sich je nach Typ der HI mit erhaltener oder reduzierter LVEF unterscheidet.

\section{Fazit für die Praxis}

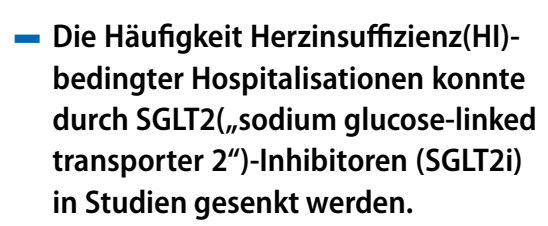


- Ein Klasseneffekt wird vermutet, es liegen aber praktisch nur zu Empagliflozin, Canagliflozin und Dapagliflozin Daten vor. Die beobachteten hohen NNT („number needed to treat") beruhen am ehesten auf geringen HI-Ereignis-Raten in der untersuchten Population.

- Subgruppenanalysen an Patienten mit bereits manifester $\mathrm{HI}$ vor Studieneinschluss ließen keine Unterschiede im Vergleich zu den anderen Studienteilnehmern erkennen, sodass vor Publikation von DAPA-HF ein HI-spezifischer Effekt unsicher erschien.

- Der HI-präventive Effekt der SGLT2i ist nach gegenwärtigem Kenntnisstand am ehesten durch einen nephroprotektiven Effekt zu erklären, da Patienten mit reduzierter Nierenfunktion in Subgruppenanalysen deutlich stärker von der Therapie profitierten als diejenigen ohne Niereninsuffizienz.

- Möglicherweise bedingt die unter SGLT2i-Therapie nachgewiesene Besserung der glomerulären Hyperfiltration eine positive Modulation der bei $\mathrm{HI}$ in schädlicher Weise überaktivierten renalen Hormonsysteme.

\section{Korrespondenzadresse}

\section{Dr. med. Andreas J. Rieth}

Kerckhoff-Klinik, Abteilung für Kardiologie, Campus Kerckhoff der Justus-Liebig-Universität Gießen

Benekestr. 2-8, 61231 Bad Nauheim,

Deutschland

a.rieth@kerckhoff-klinik.de

\section{Till Keller}

Medizinische Klinik I, Kardiologie, Justus-

Liebig-Universität Gießen

Gießen, Deutschland

Till.Keller@med.uni-giessen.de

Danksagung. Wir danken Elizabeth Martinson, PhD, für ihre redaktionelle und Hassan Bahavar für seine graphische Unterstützung.

Funding. Open Access funding enabled and organized by Projekt DEAL.

\section{Einhaltung ethischer Richtlinien}

Interessenkonflikt. C.W. Hamm gibt an, Honorare von folgenden Firmen bekommen zu haben: AstraZeneca, Bristol-Myers Squibb, Boehringer Ingelheim. C. Wanner gibt an, Honorare von folgenden Firmen bekommen zu haben: Boehringer Ingelheim, MSD, AstraZeneca, Bayer, Lilly. A.J. Rieth, V. Mitrovic und T. Keller geben an, dass kein Interessenkonflikt besteht.

Für diesen Beitrag wurden von den Autoren keine Studien an Menschen oder Tieren durchgeführt. Für die aufgeführten Studien gelten die jeweils dort angegebenen ethischen Richtlinien.

Open Access. Dieser Artikel wird unter der Creative Commons Namensnennung 4.0 International Lizenz veröffentlicht, welche die Nutzung, Vervielfältigung, Bearbeitung, Verbreitung und Wiedergabe in jeglichem Medium und Format erlaubt, sofern Sie den/die ursprünglichen Autor(en) und die Quelle ordnungsgemäß nennen, einen Link zur Creative Commons Lizenz beifügen und angeben, ob Änderungen vorgenommen wurden.

Die in diesem Artikel enthaltenen Bilder und sonstiges Drittmaterial unterliegen ebenfalls der genannten Creative Commons Lizenz, sofern sich aus der Abbildungslegende nichts anderes ergibt. Sofern das betreffende Material nicht unter der genannten Creative Commons Lizenz steht und die betreffende Handlung nicht nach gesetzlichen Vorschriften erlaubt ist, ist für die oben aufgeführten Weiterverwendungen des $\mathrm{Ma}$ terials die Einwilligung des jeweiligen Rechteinhabers einzuholen.

Weitere Details zur Lizenz entnehmen Sie bitte der Lizenzinformation auf http://creativecommons.org/ licenses/by/4.0/deed.de.

\section{Literatur}

1. Seferovic PM, Petrie MC, Filippatos GS et al (2018) Type 2 diabetes mellitus and heart failure: a position statement from the Heart Failure Association of the European Society of Cardiology. Eur J Heart Fail 20(5):853-872. https://doi.org/10. 1002/ejhf.1170

2. Ponikowski P, Voors AA, Anker SD et al (2016) 2016 ESC Guidelines for the diagnosis and treatment of acute and chronic heart failure: The Task Force for the diagnosis and treatment of acute and chronic heart failure of the European Society of Cardiology (ESC)Developed with the special contribution of the Heart Failure Association (HFA) of the ESC. Eur Heart J 37(27):2129-2200. https://doi.org/10. 1093/eurheartj/ehw128

3. Butler J, Hamo CE, Filippatos G et al (2017) The potential role and rationale for treatment of heart failure with sodium-glucose co-transporter 2 inhibitors. Eur JHeartFail. https://doi.org/10.1002/ ejhf.933

4. Moher D, Liberati A, Tetzlaff J et al (2009) Preferred reporting items for systematic reviews and metaanalyses: the PRISMA statement. PLoS Med 6(7):e1000097. https://doi.org/10.1371/journal. pmed. 1000097

5. Jorgensen L, Paludan-Muller AS, Laursen DR et al (2016) Evaluation of the Cochrane tool for assessing risk of bias in randomized clinical trials: overview of published comments and analysis of user practice in Cochrane and non-Cochrane reviews. Syst Rev 5:80. https://doi.org/10.1186/ s13643-016-0259-8

6. Zinman B, Wanner C, Lachin JM et al (2015) Empagliflozin, cardiovascular outcomes, and mortality in type 2 diabetes. $\mathrm{N}$ Engl J Med 373(22):2117-2128. https://doi.org/10.1056/ NEJMoa1504720

7. Neal B, Perkovic V, Mahaffey KW et al (2017) Canagliflozin and cardiovascular and renal events in type 2 diabetes. N Engl J Med 377(7):644-657. https://doi.org/10.1056/NEJMoa1611925

8. Wiviott SD, Raz I, Bonaca MP et al (2018) Dapagliflozin and cardiovascular outcomes in type 2 diabetes. N Engl J Med. https://doi.org/10.1056/ NEJMoa1812389

9. Cefalu WT, Leiter LA, de Bruin TW et al (2015) Dapagliflozin's effects on glycemia and cardiovascular risk factors in high-risk patients with type 2 diabetes: a 24-week, multicenter, randomized, double-blind, placebo-controlled study with a 28week extension. Diabetes Care 38(7):1218-1227. https://doi.org/10.2337/dc14-0315

10. Kosiborod M, Cavender MA, Fu AZ et al (2017) Lower risk of heart failure and death in patients initiated on sodium-glucose votransporter-2 inhibitors versus other glucose-lowering drugs: the CVD-REAL study (comparative effectiveness of cardiovascular outcomes in new users of sodium-glucose cotransporter-2 inhibitors). Circulation 136(3):249-259. https://doi.org/10.1161/ CIRCULATIONAHA.117.029190

11. Kim YG, Han SJ, Kim DJ et al (2018) Association between sodium glucose co-transporter 2 inhibitors and a reduced risk of heart failure in patients with type 2 diabetes mellitus: a realworld nationwide population-based cohort study. Cardiovasc Diabetol 17(1):91. https://doi.org/10. 1186/s12933-018-0737-5

12. Januzzi JL Jr., Butler J, Jarolim P, Sattar $N$ et al (2017) Effects of canagliflozin on cardiovascular Biomarkers in older adults with type 2 diabetes. J Am Coll Cardiol 70(6):704-712. https://doi.org/ 10.1016/j.jacc.2017.06.016

13. Kosiborod M, Gause-Nilsson I, Xu J et al (2017) Efficacy and safety of dapagliflozin in patients with type 2 diabetes and concomitant heart failure. JDiabetes Complications 31(7):1215-1221. https://doi.org/10.1016/j.jdiacomp.2017.02.001

14. Saad M, Mahmoud AN, Elgendy IY et al (2017) Cardiovascular outcomes with sodium-glucose cotransporter-2 inhibitors in patients with type Il diabetes mellitus: a meta-analysis of placebo-controlled randomized trials. Int J Cardiol 228:352-358. https://doi.org/10.1016/j.ijcard. 2016.11.181

15. Savarese G, D'Amore C, Federici M et al (2016) Effects of Dipeptidyl Peptidase 4 Inhibitors and Sodium-Glucose Linked coTransporter-2 Inhibitors on cardiovascular events in patients with type 2 diabetes mellitus: A meta-analysis. Int J Cardiol 220:595-601. https://doi.org/10.1016/j.ijcard. 2016.06.208

16. Tang H, Fang Z, Wang T et al (2016) Meta-analysis of effects of sodium-glucose cotransporter 2 inhibitors on cardiovascular outcomes and all-cause mortality among patients with type 2 diabetes mellitus. Am J Cardiol 118(11):1774-1780. https:// doi.org/10.1016/j.amjcard.2016.08.061

17. Toyama T, Neuen BL, Jun M et al (2019) Effect of SGLT2 inhibitors on cardiovascular, renal and safety outcomes in patients with type 2 diabetes mellitus and chronic kidney disease: a systematic review 
and meta-analysis. Diabetes Obes Metab. https:// doi.org/10.1111/dom.13648

18. Usman MS, Siddiqi TJ, Memon MM et al (2018) Sodium-glucose co-transporter 2 inhibitors and cardiovascular outcomes: a systematic review and meta-analysis. Eur J Prev Cardiolog 25(5):495-502. https://doi.org/10.1177/2047487318755531

19. Wu JH, Foote C, Blomster J et al (2016) Effects of sodium-glucose cotransporter-2 inhibitors on cardiovascular events, death, and major safety outcomes in adults with type 2 diabetes: a systematic review and meta-analysis. Lancet Diabetes Endocrinol 4(5):411-419. https://doi. org/10.1016/S2213-8587(16)00052-8

20. Zhang XL, Zhu QQ, Chen YH et al (2018) Cardiovascular safety, long-term noncardiovascular safety, and efficacy of sodium-glucose cotransporter 2 inhibitors in patients with type 2 diabetes mellitus: a systemic review and meta-analysis with trial sequential analysis. JAHA. https://doi.org/10. 1161/JAHA.117.007165

21. Zheng SL, Roddick AJ, Aghar-Jaffar R et al (2018) Association between use of sodium-glucose cotransporter 2 inhibitors, glucagon-like peptide 1 agonists, and dipeptidyl peptidase 4 inhibitors with all-cause mortality in patients with type 2 diabetes: a systematic review and meta-analysis. JAMA 319(15):1580-1591. https://doi.org/10. 1001/jama.2018.3024

22. Zelniker TA, Wiviott SD, Raz I et al (2019) SGLT2 inhibitors for primary and secondary prevention of cardiovascular and renal outcomes in type 2 diabetes: a systematic review and meta-analysis of cardiovascular outcome trials. Lancet 393(10166):31-39. https://doi.org/10. 1016/S0140-6736(18)32590-X

23. Kaplan A, Abidi E, El-Yazbi A et al (2018) Direct cardiovascular impact of SGLT2 inhibitors: mechanisms and effects. Heart Fail Rev 23(3):419-437. https://doi.org/10.1007/s10741-017-9665-9

24. Wanner C, Inzucchi SE, Lachin JM et al (2016) Empagliflozin and progression of kidney disease in type 2 diabetes. N Engl J Med 375(4):323-334. https://doi.org/10.1056/NEJMoa1515920

25. Vallon V, Gerasimova M, Rose MA et al (2014) SGLT2 inhibitor empagliflozin reduces renal growth and albuminuria in proportion to hyperglycemia and prevents glomerular hyperfiltration in diabetic Akita mice. Am J Physiol Renal Physiol 306(2):F194-204. https://doi.org/10.1152/ ajprenal.00520.2013

26. Hallow KM, Helmlinger G, Greasley PJ et al (2018) Why do SGLT2 inhibitors reduce heart failure hospitalization? A differential volume regulation hypothesis. Diabetes Obes Metab 20(3):479-487. https://doi.org/10.1111/dom.13126

27. Inzucchi SE, Zinman B, Fitchett D et al (2018) How does empagliflozin reduce cardiovascular mortality? Insights from a mediation analysis of the EMPA-REG OUTCOME trial. Diabetes Care 41(2):356-363. https://doi.org/10.2337/dc171096

28. McMurray JJV, Solomon SD, Inzucchi SE et al (2019) Dapagliflozin in patients with heart failure and reduced ejection fraction. N Engl J Med. https:// doi.org/10.1056/NEJMoa1911303

29. SPRINT Research Group, Wright JT Jr., Williamson JD, Whelton PK et al (2015) A randomized trial of intensive versus standard blood-pressure control. N Engl J Med 373(22):2103-2116. https:// doi.org/10.1056/NEJMoa1511939

30. Nassif M, Kosiborod M (2018) Effect of glucoselowering therapies on heart failure. Nat Rev Cardio 15(5):282-291. https://doi.org/10.1038/nrcardio. 2017.211 